\title{
Novel wire-guided fine-gauge bougie dilator for transpapillary or endoscopic ultra- sonography-guided biliary drainage
}

Endoscopic management of biliary stricture generally requires dilation using devices such as an endoscopic retrograde cholangiopancreatography (ERCP) balloon dilation catheter before stenting [1]. Endoscopic ultrasonography (EUS)guided biliary drainage (EUS-BD) also requires fistula dilation before stenting. Recently, ultraslim balloon catheters [1] and diathermic dilators [2] have also been developed as dilation devices. These devices must be wire-guided, coaxial with the guidewire, fine-gauge, and sufficiently stiff.

Herein, we present two patients who successfully underwent biliary dilation using a novel wire-guided fine-gauge bougie dilator (ES dilator soft type; Zeon Medical Inc., Tokyo, Japan) (\Fig. 1 and - Fig.2) for transpapillary drainage and EUS-BD.

The first patient was a 79-year-old man who was admitted with obstructive jaundice having undergone placement of self-expandable metal stents (SEMSs) for perihilar bile duct cancer 5 months previously. An ERCP showed occlusion of the SEMSs ( $>$ Fig. 3 a). First, a 0.025-inch hard-type guidewire (VisiGlide 2; Olympus, Tokyo, Japan) was advanced across the occluded SEMSs. A tapered ERCP catheter and a dilation catheter (SBDC-6; Cook Japan, Tokyo, Japan) could not be passed through the stricture ( $\vee$ Video 1 ). The novel dilator was then inserted, resulting in successful passage through the

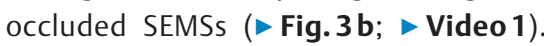
Finally, an uncovered SEMS was placed without any complications.

The second patient was an 85-year-old man who was admitted with obstructive jaundice and a history of total gastrectomy and Roux-en-Y reconstruction for gastric cancer 21 years previously. A computed tomography (CT) scan showed an ampullary tumor and treatment by EUS-BD was selected. Firstly, B3 was punctured with a 19-gauge needle via the jejunum and a 0.025 -inch hard-

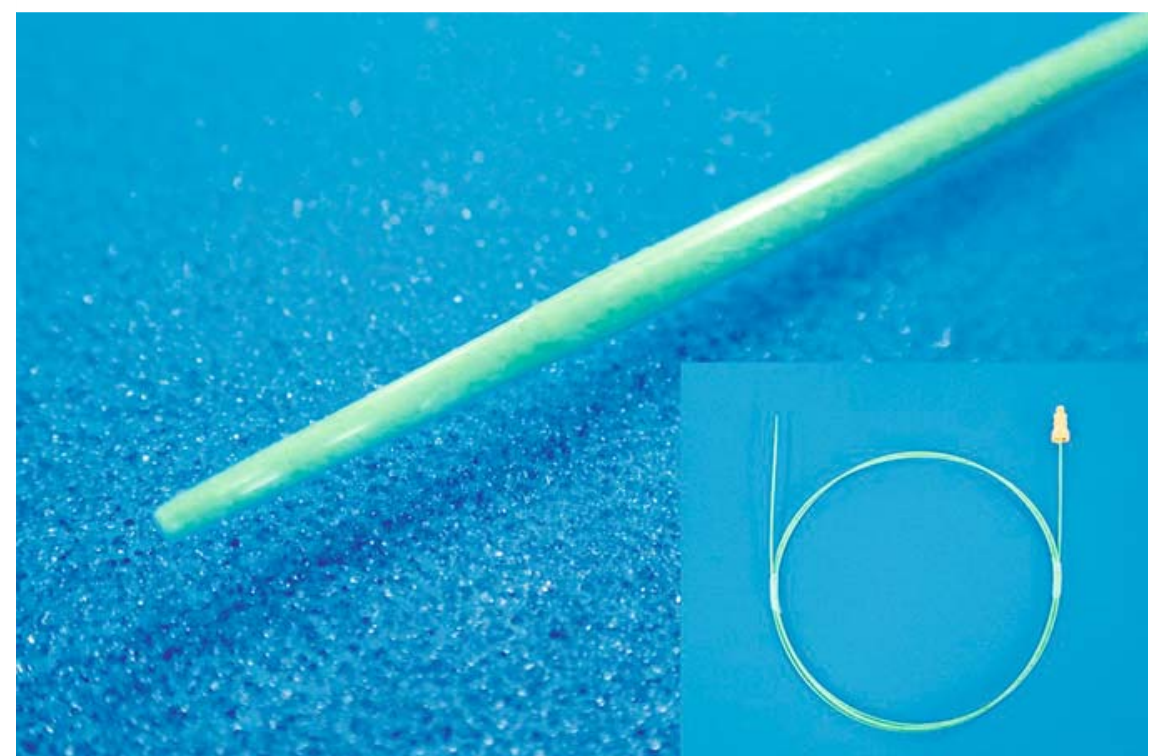

- Fig. 1 The tip of the novel fine-gauge bougie dilator (ES dilator soft type; Zeon Medical Inc., Tokyo, Japan) is 3.2-Fr in diameter, tapered, and coaxial with the 0.035 -inch guidewire, and has a radiopaque marker to help during insertion. Its maximum diameter is 7.4-Fr. Inset: Image of the whole dilator.

type guidewire (VisiGlide 2; Olympus) was placed. A tapered ERCP catheter was tried without success to dilate the fistula. Subsequently, dilation with the novel dilator was attempted, and this was successfully inserted into the intrahepatic bile duct ( $>$ Fig.4; $>$ Video 2). Finally, EUS-guided antegrade stenting

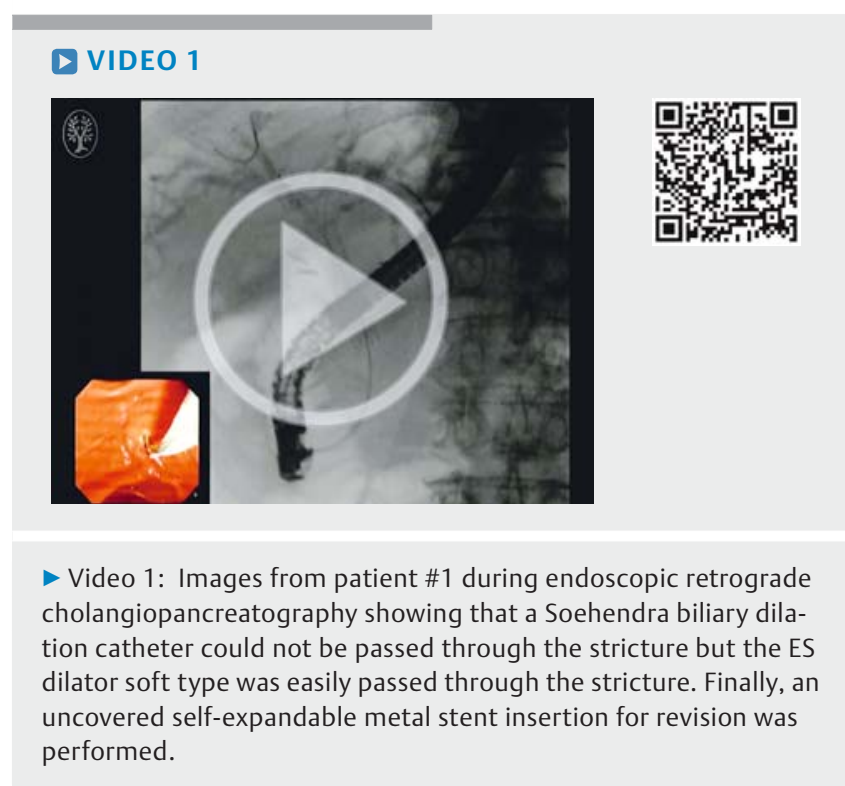




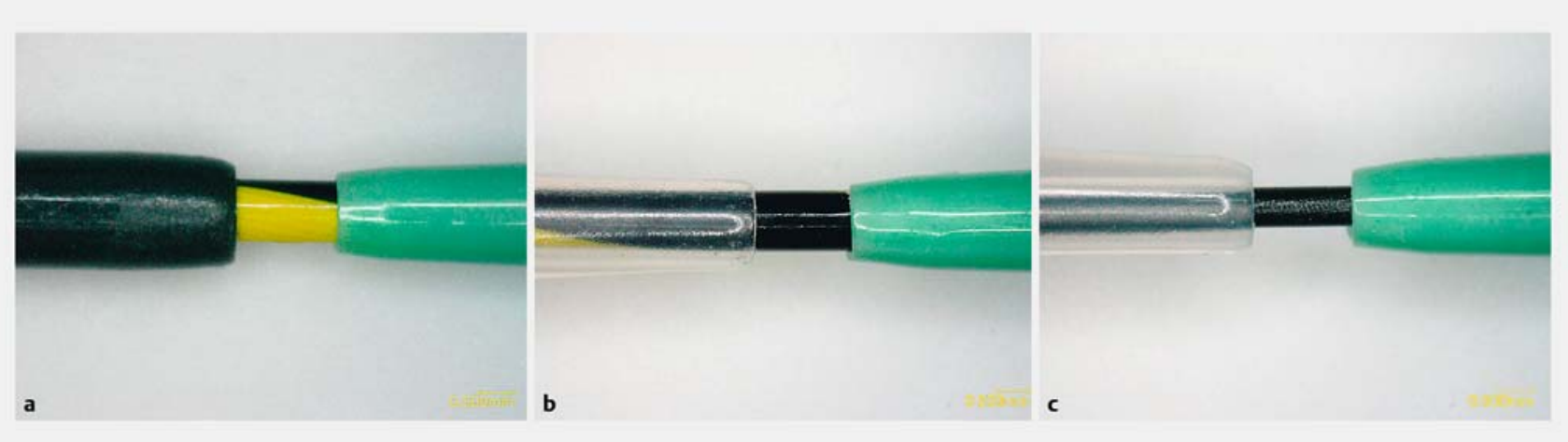

- Fig. 2 The novel fine-gauge bougie dilator (ES dilator soft type; 3.2-Fr tip; Zeon Medical Inc., Tokyo, Japan; right side in all images) is compared with: a a conventional bougie dilator (SBDC-6; 4-Fr tip and maximum diameter of 6-Fr; Cook Japan, Tokyo, Japan) shown placed over a 0.035-inch guidewire; $\mathbf{b}$ an ultraslim balloon catheter (ZARA EPBD balloon; 3.1-Fr tip and maximum diameter of 6.3-Fr; Century Medical Inc., Tokyo, Japan) shown placed over a 0.035-inch guidewire; c an ultraslim balloon catheter (REN biliary dilation catheter; 3-Fr tip and maximum diameter of 6.4-Fr; Kaneka Corporation, Osaka, Japan) shown placed over a 0.025 -inch guidewire. The ES dilator and ZARA EPBD balloon are excellent for having minimal difference in caliber between the guidewire and the catheter.

was performed without any complications ( $\triangleright$ Video 2 ).

The novel wire-guided fine-gauge bougie dilator is useful for both transpapillary and fistula dilation in EUS-BD owing to its ideal thickness and stiffness.

Endoscopy_UCTN_Code_TTT_1AS_2AD

\section{Competing interests}

The novel bougie dilator has been developed through collaborative research between Dr. Kawakami and Zeon Medical Inc., Tokyo, Japan. Dr. Kawakami is a consultant and gives lectures for the Zeon Medical Inc. The authors declare no conflict of interests for this article.

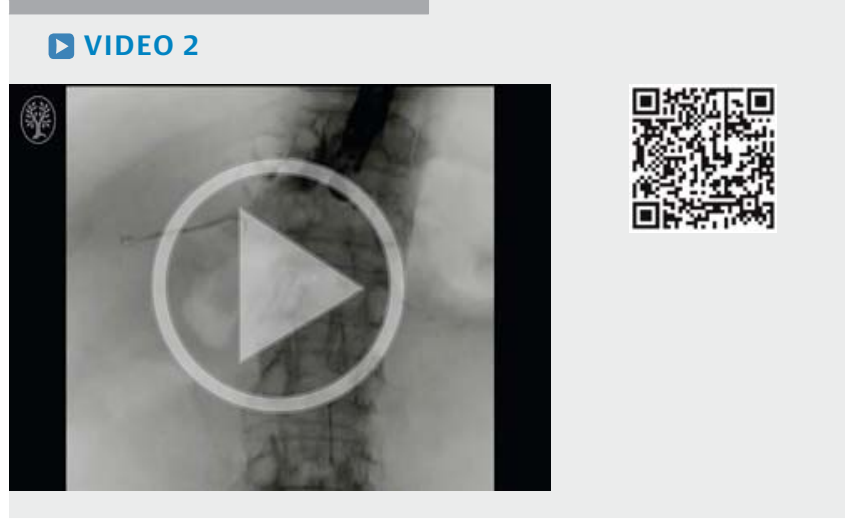

Video 2: Images from patient \#2 during endoscopic ultrasonography (EUS)-guided biliary drainage showing a guidewire placed in the intrahepatic bile duct after puncture of B3 with a 19-gauge needle. A tapered endoscopic retrograde cholangiopancreatography (ERCP) catheter could not be passed through the stricture; however, the ES dilator soft type was inserted with ease. It was then possible to pass a tapered ERCP catheter through the distal biliary stricture and finally EUS-guided antegrade stenting was performed for the distal biliary obstruction. 

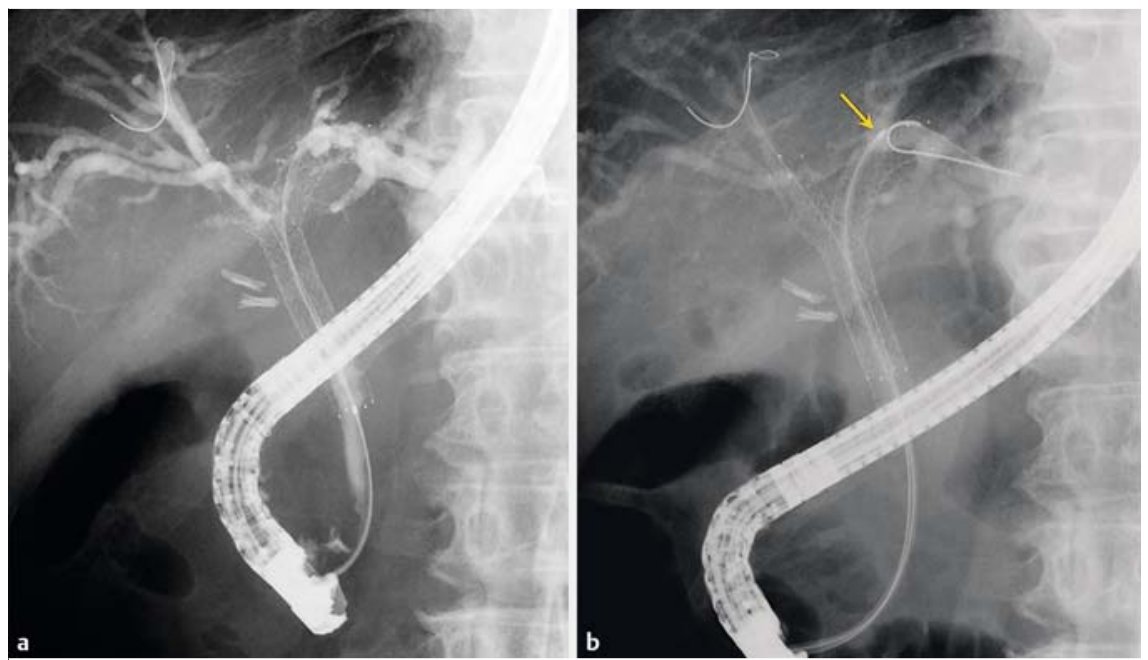

- Fig. 3 Radiographic images from patient \#1 showing: a recurrent perihilar biliary obstruction after multistenting in a stent-in-stent fashion; $\mathbf{b}$ the ES dilator soft type (arrow) that has been passed through the refractory biliary stricture.

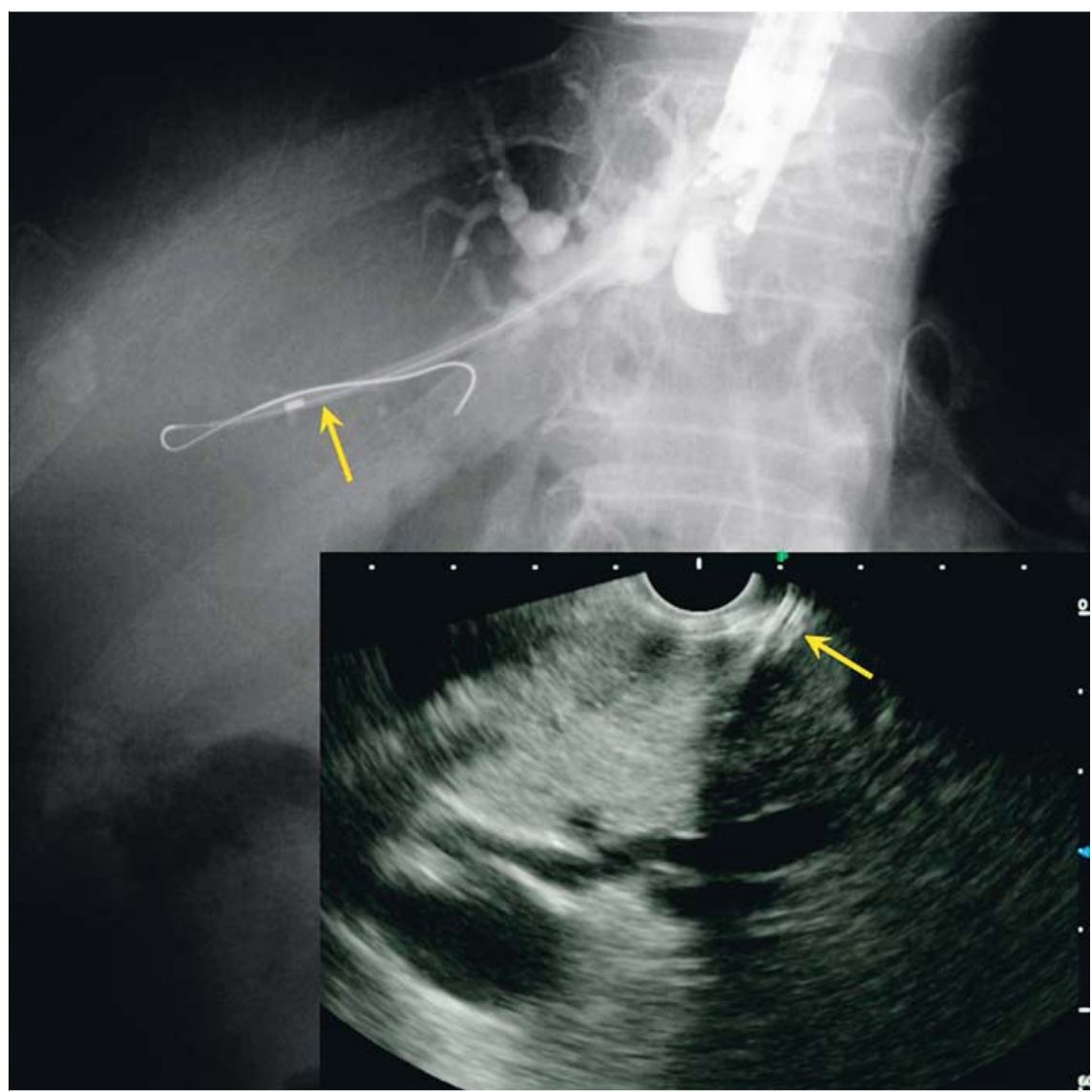

Fig. 4 Radiographic image from patient \#2 showing the ES dilator soft type (arrow) that was successfully advanced through the jejunal wall and intrahepatic bile duct after a failed attempt at fistula dilation using a tapered endoscopic retrograde cholangiopancreatography catheter. Inset: Endoscopic ultrasonography view showing the ES dilator soft type (arrow).
The Authors

Hiroshi Kawakami, Yoshimasa Kubota

Department of Gastroenterology and

Hepatology, University of Miyazaki and Center for Digestive Disease, University of Miyazaki Hospital, Miyazaki, Japan

\section{Corresponding author}

\section{Hiroshi Kawakami, MD, PhD}

Department of Gastroenterology and

Hepatology, University of Miyazaki,

Center for Digestive Disease, University of Miyazaki Hospital, 5200, Kihara, Kiyotake, Miyazaki 889-1692, Japan

Fax: +81-985-859802

hiropon@med.miyazaki-u.ac.jp

\section{References}

[1] Kawakami H, Kuwatani M, Kawakubo K et al. Transpapillary dilation of refractory severe biliary stricture or main pancreatic duct by using a wire-guided diathermic dilator (with video). Gastrointest Endosc 2014; 79: 1-3

[2] Ogura T, Takagi W, Onda S et al. Endoscopic ultrasound-guided biliary drainage with a novel fine-gauge balloon catheter: simplified technique using a coaxial guidewire. Endoscopy 2015; 47: E573 -E574

\section{Bibliography}

DOI http://dx.doi.org/10.1055/s-0042-124498

Endoscopy 2017; 49: E75-E77

(c) Georg Thieme Verlag KC

Stuttgart · New York

ISSN 0013-726X 